\title{
Exploring Multiple Usability Perspectives
}

\author{
Tobias Uldall-Espersen \\ Department of computing, University of Copenhagen, Universitetsparken 1, \\ DK-2100 Copenhagen, Denmark \\ tobiasue@diku.dk
}

\begin{abstract}
Industrial usability work often fails to produce the expected impact on software products even though significant resources have been used on uncovering problems and suggesting improvements. So, it seems that feedback from industrial usability work lacks persuasiveness, i.e. it fails to convince the key stakeholders that actions need to be taken. This study reports from interviews with 26 stakeholders in software development projects. Our data suggests that the interviewees address usability using different perspectives and based on our observations we describe five such perspectives. Further, we discuss how applying different usability perspectives might inform the persuasiveness of usability work.
\end{abstract}

\section{Introduction}

One important problem when developing software is that usability work does not sufficiently inform software development even though a large number of usability issues are identified. This problem is in the literature described as lack of designchange effectiveness [4], lack of downstream utility [2], or lack of impact [1], and can partly be explained by lack of persuasive power [4] in the usability feedback. Recent studies show that a large number of usability issues are known to stakeholders prior to usability evaluations are conducted $[1,3,5]$, and this suggests that feedback given to stakeholders are not adequate. In this paper we suggest an approach to explore and possibly increase adequacy and persuasiveness of feedback from usability work. In a resent paper [6] we argue, that usability as defined in ISO 9241-11 can be oriented towards (1) the user interface or user interests, and/or (2) the organization or other stakeholders. Here, we expand this approach by arguing that different usability perspectives are in play when developing software. Data originates from an ongoing interview study involving 26 stakeholders from six industrial software development projects in Denmark. Our observations are extracted using grounded theory (see [6]). The limited space in this paper makes it impossible to fully document our findings, but we aim at describing five frequently observed significant usability perspectives.

\section{The Five Usability Perspectives}

\subsection{The Interaction Object Usability Perspective}

Interaction object usability concerns whether users are able to successfully perform isolated interactions with user interface objects in the product. We saw how 
consistency was a concern using this perspective, and how standards and guidelines informed the visual design and interaction design of user interface objects. We also saw how developers were given considerable freedom regarding interaction object usability. Our data suggests that interaction object usability interplays with the applied technology (i.e. hardware, software and infrastructure), and thus that technology can inform the possibilities to produce usable software. For example we saw how a shift to wireless technology in a mobile sales support application significantly changed the usability of input fields. Online data validation was introduced reducing the amount of errors in data, but increased response time when entering data in the system. Furthermore we saw how development standards informed interaction object usability, e.g. by disallowing use of "mouse over" events on buttons, which in one case was requested by the designer.

Our data shows how interaction object usability was handled through use of a number of the traditional usability evaluation methods, such as user tests, expert evaluations, and use of guidelines or standards. Using this perspective our findings suggest that we need to take both the users and the context of use into consideration, and especially the users skills and familiarity with the technology seems important.

\subsection{The Task Usability Perspective}

Task usability concerns whether the users are able to complete single tasks, i.e. fulfill a (sub) goal through a combination of interactions with user interface objects. We observed how some tasks received high level of attention when implementing new software in organizations and that the level of attention dedicated to individual tasks varied considerably. Simplicity and completeness of tasks received high attentions. Simplicity means that users only need limited knowledge related to the task to complete it, and lack of simplicity was to some degree counterbalanced through user education. Completeness means that tasks should embrace and successfully complete the corresponding work process. We observed how technology informed task usability since different technologies provide different possibilities and solutions. Furthermore we observed how techniques determining task flows informed task usability and the motivation to evaluate task usability. For example we saw how a strong process oriented development approach supported developing tasks that covered the entire process, but with an ineffectively implemented design.

Evaluating task usability required knowledge about the tasks, the users, the domain, and the context of use and was often done using different variations of user testing. Also, role-plays showed to be useful when testing tasks involving interaction between humans while using the system, e.g. in sales or interview situations.

\subsection{The Product Usability Perspective}

Product usability concerns whether the product supports the users in reaching the coherent set of goals with efficiency, effectiveness and satisfaction. We observed how product usability was depending on whether the product provided flexibility, consistency, and completeness during usage.

Product usability seems to play a more important role in products with complex user interaction or products with an explorative nature compared with simple 
products. Task usability concerns having a straight way to reach a specified goal. In contrast product usability concerns interplay between different parts of the product allowing numerous roads to reach important goals. We observed how the degrees of freedom of use made it hard to predict and evaluate product usability, since it required a thorough overview of the product and its usage. Knowing the specified tasks is not sufficient and a very open approach is needed. Furthermore our data shows that field observations were neither extensively nor widely used, but occasionally used in small scale.

\subsection{The Context of Use Usability Perspective}

Context of use usability concerns to what extent use of the system, possibly interplaying with other systems, in the actual context of use is effective, efficient, and satisfactory. Consistency across IT-systems and/or manual systems, systems integration, and inter-human relations during use of the system were important factors that influenced context of use usability. We saw how these factors had significant influence on business performance. Further we saw how users in complex work situations worked with and combined data from various systems, also informing context of use usability.

Context of use usability was rarely addressed systematically in the cases in our study. This could be explained by the fact that context of use experts only superficially were involved in the usability work. Also, systems interplay issues need to be addressed across projects rather than within projects, which increases the complexity of usability work using this perspective. Furthermore, we saw how important context of use usability issues were known from earlier versions of systems, and how workshops with context of use experts were used to address such issues in the early phases of the projects.

\subsection{The Enterprise Usability Perspective}

Enterprise usability concerns to what extent goals of the enterprise are fulfilled effectively, efficiently, and satisfactorily through use of the system. This concern is not necessarily related to the users of the systems, but rather depends on whether use of the system informs or is informed by the enterprise. Enterprise usability seemed informed by three conditions: First, we saw how visions combined with ITdevelopment projects supported stakeholders in working towards common goals rather than individual goals. Second, we saw how systems integration supported utilizing information across the enterprise and enabled support to related work processes in other departments. We also saw how failing to integrate systems could jeopardize the success of a project. Third, we saw how consistency and completeness in processes across the enterprise supported that individual completed tasks together made out a coherent environment.

In our study enterprise usability was addressed by involving key stakeholders in the initial phase, e.g. through workshops, and by maintaining their involvement throughout the process. Since enterprise usability concerns issues from all over the enterprise, it seems necessary to involve the widest range of stakeholders and filter the information afterward rather than limiting the number of involved stakeholders. 


\section{Discussion}

In an ongoing interview study covering six software development projects, five perspectives on usability was observed. In relation to the conducted usability work in the projects, the observed perspectives had a significant practical importance. They rose from different approaches to usability among the stakeholders and revealed both conflicting and coherent interests regarding identified usability issues. Our data suggests that usability issues related to different perspective have different properties, and studying these properties will be objects for further research. We will do this by addressing the following hypotheses:

- Persuasiveness of usability issues increases if different usability perspectives point to the same solution. Persuasiveness decreases if they point to conflicting solutions.

- Different usability perspectives appeal to different stakeholders and inform business value differently.

- Different usability perspectives are relevant at different stages of the software development process.

At the time of this writing we are looking for everyday examples from industry and related research to support our observations. One example comes from the eCommerce sector where business revenue and user experience are closely tied together bringing multiple usability perspectives into play. Another example comes from development of safety critical systems where usability problems can have severe consequences for the entire enterprise. Thus, multiple perspectives on usability could help ensuring that no stakeholders suffer from lack of usability.

In the cases we have studied, the multiple perspectives of usability were not treated systematically. Even in current research and in the state-of-the-art techniques, we rarely see such perspectives addressed and the literature fails to support practitioners in working with and understanding the perspectives. Consequently, the outcome of usability work could continue to be inadequate and non-persuasive constituting a significant risk of failure when developing industrial software.

\section{Acknowledgement}

This work is part of the USE-project (Usability Evaluation \& Software Design) founded by the Danish Research Agency through the NABIIT Programme Committee (Grant no. 2106-04-0022). I thank Erik Frøkjær for rewarding discussions developing the ideas to this paper.

\section{References}

1. Følstad, A.: Work-Domaine Experts As Evaluators: Usability Inspection of DomainSpecific Work-Support Systems. IJHCI 22(3), 217-245 (2007)

2. Hartson, H.R., Andre, T.S., Williges, R.C.: Criteria for Evaluation Usability Evaluation Methods. IJHCI 13, 373-410 (2001) 
3. Hornbæk, K., Frøkjær, E.: Comparing Usability Problems and Redesign Proposals As Input to Practical Systems Development, pp. 391-400. ACM Press, New York (2005)

4. John, B.E., Marks, S.J.: Tracking the Effectiveness of Usability Evaluation Methods. BIT 16, 188-202 (1997)

5. Molich, R., Dumas, J.S.: Comparetive Usability Evaluation (CUE-4). BIT, 1-19 (2007)

6. Uldall-Espersen, T., Frøkjær, E.: Usability and Software Development: Roles of the Stakeholders. In: HCI International, Springer, Heidelberg (2007) 